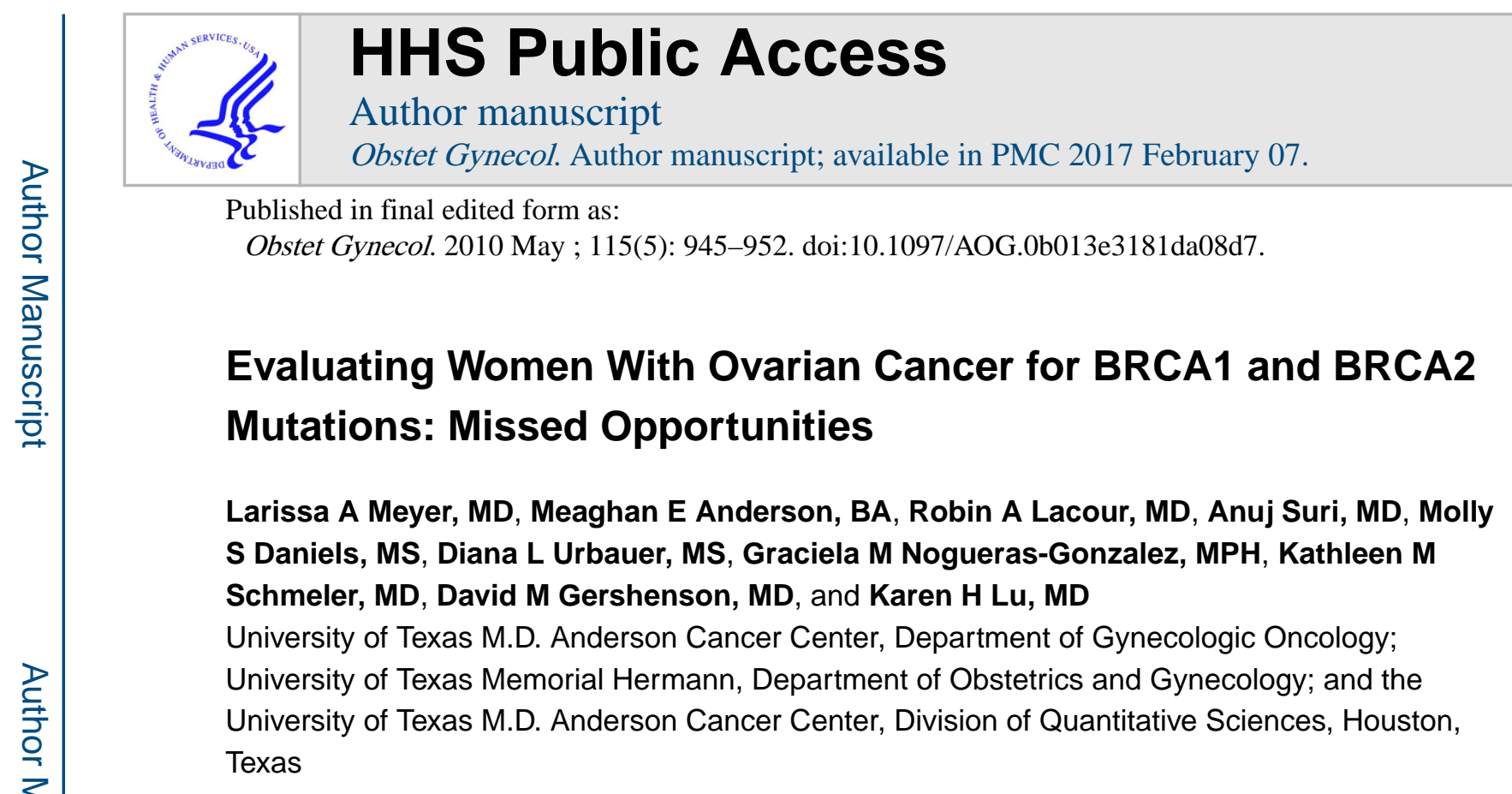

\title{
Abstract
}

OBJECTIVE-To estimate the incidence of genetic counseling referral for ovarian cancer patients who are at substantial risk for a BRCA1 or BRCA2 mutation.

\begin{abstract}
METHODS-An analysis was performed of new ovarian cancer patients who were seen at a comprehensive cancer center from January 1, 1999, through December 31, 2007. Patients at substantial (more than 20-25\%) risk for a BRCA1 or BRCA2 mutation were identified and records reviewed for referral to genetic counseling. Time to referral was estimated using the Kaplan-Meier method.
\end{abstract}

RESULTS-A total of 3,765 epithelial ovarian cancer patients were seen during the 9-year period. On average, $23.8 \%$ of patients met substantial-risk criteria for BRCA mutations. In 1999, only $12 \%$ of patients at substantial-risk were referred. Referral improved over time with $48 \%$ referred in $2007(\mathrm{P}<.001)$. Newly diagnosed patients were more often referred for genetic counseling than new patients with recurrent disease or those seen as second opinions. AfricanAmerican women meeting substantial-risk criteria were less likely to be referred than were white or Hispanic women $(\mathrm{P}=.009)$.

CONCLUSION-Although dictated family history was accurate, interpretation of risk for BRCA1 or BRCA 2 mutations and subsequent referral to genetic counseling was poor. Although there was significant improvement over time, $50 \%$ of substantial-risk patients still were missed. Systematic efforts to identify those ovarian cancer patients at substantial risk for a BRCA1 or BRCA2 are necessary.

\section{LEVEL OF EVIDENCE-III}

Clinical genetic testing for germline mutations in BRCA1 and BRCA2, the genes that account for the majority of hereditary breast and ovarian cancer families, has been available

Corresponding author: Karen H. Lu, MD, PO Box 301439, Houston, TX 77230-1439; khlu@mdanderson.org.

Presented at the 2009 Society of Gynecologic Oncologists Annual Meeting, February 5-8, 2009, San Antonio, Texas. Also presented in part at the 2008 American Society of Clinical Oncology Annual Meeting, May 30-June 3, 2008, Chicago, Illinois, and at the Western Society of Gynecologist Oncologists/Felix Rutledge Society Joint Annual Meeting, June 11-14, 2008, Sonoma, California. 
for more than a decade. [1,2] In the general population, the frequency of BRCA1 or BRCA2 mutations is estimated to be 1 in 300-500. [3-5] Among breast cancer patients, approximately $5 \%$ have germline mutations in BRCA1 or BRCA2. [6] Recent populationbased studies of ovarian cancer patients found that $11.7 \%-15.3 \%$ had germline mutations in BRCA1 or BRCA2. [7,8]

Multiple prediction models have been developed to estimate the likelihood that a particular individual has a germline mutation in BRCA1 or BRCA2. [9-12] Published consensus guidelines also help guide clinicians to identify individuals at increased risk for BRCA1 or BRCA2 mutations. [13-15] Consistent with criteria in the prediction models, the American College of Obstetricians and Gynecologists Practice Bulletin on Hereditary Breast and Ovarian Cancer syndrome defines criteria for ovarian cancer patients who are at substantial (more than 20-25\%) risk for having a BRCA1 or BRCA2 mutation who should be referred for genetic counseling. [15] These include women with ovarian cancer who 1) also have a personal history of breast cancer; 2) are of Ashkenazi Jewish ancestry, or 3) have a close relative with breast cancer age 50 or younger or ovarian cancer at any age. [9-14]

In recent years, it has become clear that there are important reasons to focus genetic testing efforts on the cancer patient. First, directing genetic testing efforts toward cancer patients rather than unaffected individuals is an efficient means to identify the specific deleterious mutation in a family. [16-18] Once the specific mutation is identified, unaffected family members can be offered accurate genetic testing just for the known mutation. In this setting, both positive and negative genetic test results are interpretable and informative. Although genetic testing in the past has not had an effect on the treatment of ovarian cancer patients, clinical trials of poly ADP ribose polymerase inhibitors currently are underway specifically for breast and ovarian cancer patients with BRCA1 or BRCA2 mutations, and different therapeutic regimens may be prescribed based on a patient's genetic test results. In addition, BRCA status has prognostic significance for ovarian cancer patients. [19-23]

Given the importance of identifying ovarian cancer patients who are highly likely to carry a BRCA1 or BRCA2 mutation, the question remains: "How successful are physicians at identifying these individuals?" The purpose of this study was to estimate the incidence of referral of new ovarian cancer patients who had a substantial (more than 20-25\%) risk for having a BRCA1 or BRCA2 mutation. We hypothesized that even at a tertiary cancer center, ovarian cancer patients who had a personal or family history highly suggestive for a BRCA1 or BRCA2 mutation were not adequately being identified and referred for genetic counseling.

\section{MATERIALS AND METHODS}

Approval was obtained from the University of Texas M.D. Anderson Cancer Center's institutional review board. All patients with a diagnosis of ovarian, fallopian tube or primary peritoneal cancer registered as new patients at our institution from January 1, 1999, to December 31, 2007, were identified from our institutional tumor registry. All patients with epithelial ovarian, fallopian tube or primary peritoneal cancer who were seen as a new patient by either gynecologic or medical oncologists and who had a full initial history and 
physical in their medical record during the study period were included. Patients with nonepithelial ovarian cancer, tumors of low malignant potential, and patients who did not have a full evaluation or who were seen for a malignancy other than ovarian cancer were excluded. The initial history and physical in the medical record was reviewed for family history and personal cancer history information. When available, this same data were abstracted from a personal history data questionnaire filled out by the patient. Clinical and demographic information was collected.

Individuals were identified who had personal or family histories meeting the following substantial (more than 20-25\%) risk criteria for having a BRCA1 or BRCA2 mutation: 1) women with a personal history of both breast and ovarian cancers (peritoneal and fallopian tube cancers are included), 2) women with ovarian cancer who are of Ashkenazi Jewish ancestry, or 3) women with ovarian cancer and a close relative (first-, second-, or thirddegree relative) with breast cancer age 50 or younger or ovarian cancer at any age. [14,15] A documented discussion recommending genetic counseling or evidence of a requisition or visit with the genetic counselor counted as a genetic counseling referral for the purposes of this study. If documentation from the initial visits did not acknowledge a discussion regarding genetic counseling, then the medical records from all subsequent patient visits at our institution were reviewed for evidence of referral to genetic counseling at a later date. Individuals who were diagnosed with a BRCA1 or BRCA2 mutation before their initial visit to our institution were included in the analysis as a successful referral to genetic counseling.

Descriptive statistics, Cochran-Armitage trend test, Fisher's exact, $t$ test, log-rank test, and Kappa statistics with an a-level of 0.05 were utilized for analysis. Time to referral was estimated using the Kaplan-Meier method. The date of the first visit to the institution for ovarian cancer care was used as the starting point and the date of referral was determined by a clinician dictating discussion for need of genetic counseling or by the date of the genetic counseling consult request. Multivariate logistic regression analyses were performed. In addition to the full model, backwards selection techniques were employed only keeping terms that were statistically significant with $\mathrm{P}<.10$.

\section{RESULTS}

The medical records of 5,208 women with a diagnosis of ovarian cancer registered as new patients at our institution between January 1, 1999, and December 31, 2007, were reviewed. A total of 3,765 women had invasive epithelial ovarian cancer and were included in the analysis. Summary demographic information is available in Table 1 . The mean age of the women was 56 years (standard deviation \pm 11.8 years). The majority of patients had highgrade, serous ovarian cancer (Table 1).

The number of patients with dictated family history in the initial history and physical increased over time from $85.6 \%$ in 1999 to $95.9 \%$ in 2007 ( $\mathrm{P}<.001$ ). Family history information was also available from the self-reported patient history questionnaire for 2,156 $(57.3 \%)$ women. Agreement between the self-reported questionnaire and dictated family history was high ( $\kappa 0.86,95 \%$ CI $0.84-0.89$ ). The proportion of patients meeting substantial BRCA1 or BRCA2 risk criteria was relatively stable over the 9-year study period, ranging 
from $20.2-28.8 \%$. On average over the study period, $23.8 \%$ of patients met substantial risk criteria for a BRCA1 or BRCA2 mutation (Table 2).

In 1999 , only $12 \%$ of women with ovarian cancer at substantial risk (more than $20-25 \%$ chance of having a BRCA1 or BRCA2 mutation) were referred for genetic counseling. There was a significant improvement in the proportion of substantial risk patients referred over time with $48 \%$ referred in 2007 ( $\mathrm{P}<.001$ ) (Fig. 1). There was a 28\% increased chance of referral per year when compared with the previous year (OR 1.28, 95\% CI 1.17-1.40). Although the proportion of patients at substantial risk for a BRCA1 or BRCA2 mutation who were referred immediately improved significantly $(\mathrm{P}<.001)$ over the 9-year study period, the median time to referral was more than 3 years across all study periods (Fig. 2).

Out of 3,765 ovarian cancer patients, 2,793 (74.2\%) were treated at an outside institution for ovarian cancer care before presentation to our institution. Individuals who were diagnosed with a BRCA1 or BRCA2 mutation before their initial visit to our institution were included in the analysis as a successful referral. In total over the 9-year study period, 276 of 896 patients $(30.8 \%$ ) who met substantial risk (more than $20-25 \%$ chance of having a BRCA1 or BRCA2 mutation) criteria were referred for genetic counseling. Of those 276, 92 (33.3\%) patients presented as new patients to our institution having been previously referred for genetic counseling.

In a multivariate analysis performed to predict which patients were referred out of the subset of patients who met criteria for being at substantial risk (more than 20-25\% chance of having a BRCA1 or BRCA2 mutation), personal history of breast cancer, positive family history of either premenopausal breast or ovarian cancer at any age, and Jewish religion were associated with an increased odds of referral (Table 3). Other variables with a significantly increased odds of referral include year of initial visit, age at first visit and grade 3 disease.

There was a statistically significant difference in patient type and race between ovarian cancer patients meeting substantial risk who were referred and not referred. Newly diagnosed patients were more often referred for genetic counseling than new patients who presented with recurrent disease or patients seen for a second opinion $(\mathrm{P}<.001)$ (Fig. 3). Ovarian cancer patients who transferred their care with recurrent disease were $56 \%$ less likely to be referred than women who were being seen with newly diagnosed disease. Those patients with recurrent disease who were seen for a second opinion were $77 \%$ less likely to be referred (Table 3). African-American women with ovarian cancer who met substantial risk criteria were less likely to be referred for genetic counseling than white women (OR $0.25,95 \%$ CI 0.09-0.70) (Table 3).

During the 9-year time period, 242 ovarian cancer patients from this cohort were seen for genetic counseling. Of those, 208 chose to undergo germline DNA testing for BRCA1 and BRCA2 mutations. Of the women who underwent genetic testing, 102 (49\%) were found to have a deleterious mutation, 78 with a BRCA1 mutation and 24 with a BRCA2 mutation. 


\section{DISCUSSION}

Our study suggests that physicians are missing important opportunities to identify ovarian cancer patients with germline BRCA1 and BRCA2 mutations. Interestingly, we found that health care providers were dictating family history in the initial history and physical in a large majority of patients, and there was agreement between the dictated family history and the self-reported questionnaire. However, referral of those high-risk patients to genetic counseling was poor in 1999, and, although improved in 2007, was still below 50\%. In addition, we found that African-American women with ovarian cancer who met the substantial risk criteria were less likely to be referred for genetic counseling than white or Hispanic women.

The first step to improving the delivery of health care is to understand and define the deficit. Although this analysis is limited in that it is a single institution study, more than half of the patients were seen elsewhere before being seen as new patients at our tertiary care cancer center. Given the availability of cancer genetics at a tertiary care cancer center, we believe that it is possible that the incidence of referral is even lower in a community setting.

At our institution, the process by which cancer genetic counseling services are provided has evolved. During this study period, we did not have a systematic method for identifying those ovarian cancer patients with high-risk criteria. Individual clinicians had different practice patterns for referring patients to genetic counseling. This lack of a systematic approach likely resulted in our low incidence of referral. However in 2006, genetic counselors were integrated into the gynecology department. Now patients are seen for genetic counseling appointments in the same clinic space where they receive their routine oncology care. Appointments are coordinated with other clinic visits and counselors are available for urgent consults in the outpatient and inpatient setting.

As the medical literature has evolved over the past 10 years, physicians have become more aware of the prevalence of BRCA mutations in ovarian cancer patients. This awareness has likely led to an increased number of referrals. Published guidelines from groups such as the American College of Obstetricians and Gynecologists have been developed which outline criteria for hereditary cancer risk assessment and genetic testing for germline BRCA1 and BRCA2 mutations. [13-15] In addition, patients and family members may be requesting BRCA testing more frequently as there has been increased coverage of BRCA mutation testing by the lay press. However, even in recent years our incidence of referral for substantial risk patients has been surprisingly low.

It is not clear when is the best time to refer ovarian cancer patients to genetic counseling. Family history and past medical history are routinely obtained at the initial visit and may not be reviewed during future focused visits. A discussion regarding the need for genetic counseling is rarely an emergency and is thus, often postponed and then overlooked. Our findings suggest that cancer patients at increased risk for a hereditary cancer syndrome were less likely to be referred as time elapsed after their first visit. Patients seen as new patients in the setting of recurrent disease or for second opinions were also less likely to be referred than patients presenting with a new diagnosis of ovarian cancer. We believe that this may be 
a result of the physician having a more focused approach to those patients and not integrating the family or personal past medical history in the same way as they would with a newly diagnosed ovarian cancer patient. Regardless of when each practice or physician chooses to screen patients for risk of hereditary cancer syndromes, it is most likely to be successful if performed in a systematic fashion.

Evidence suggests that those who already have cancer may be more willing to have genetic testing. [24] The traditional barriers to genetic counseling such as financial concerns $[17,24,25,26]$ and the fear of genetic discrimination $[17,27,28]$ may be decreased when cancer patients are tested. In addition, testing the cancer patient for a germline BRCA mutation is more likely to be covered by insurance. [29] Finally, the fear of genetic discrimination may be less of an issue in individuals with cancer as their diagnosis trumps any theoretical predictive risk implied by a germline mutation. Indeed, one recent study found that $89 \%$ of ovarian cancer patients would be willing to have genetic testing for BRCA mutations if it would directly affect their cancer therapy and $87 \%$ would be willing even if it was solely to benefit family members. [30]

How many ovarian cancer patients attend genetic counseling after referral, how many decide to undergo testing for germline BRCA1 and BRCA2 mutations, and then ultimately, how many test positive for mutations were not the primary aim of this current analysis. In this study we chose to focus on the physician's role of identifying which patients fulfill published guidelines for hereditary cancer risk assessment as it is the first step in the process and is the only step that is under the full and direct control of the health-care provider. Improving the number of appropriate referrals is an important aspect of cancer prevention efforts. Indeed, $49 \%$ of ovarian cancer patients who chose to undergo testing were found to have a deleterious mutation in BRCA1 or BRCA2. This high incidence of positive tests suggests that applying published guidelines such as the American College of Obstetricians and Gynecologists Practice Bulletin on Hereditary Breast and Ovarian Cancer syndrome [15] to clinical practice can successfully focus genetic testing efforts in a high-risk population.

In conclusion, failure to identify cancer patients who have an inherited cancer predisposition has many important implications, and standardized interventions are clearly needed to improve the incidence of appropriate referrals of cancer patients at risk for hereditary cancers who may benefit from genetic testing. Given the current and future therapeutic implications, greater attention to developing a systematic process of identifying cancer patients with hereditary cancer syndromes is needed. At-risk individuals should be referred to genetic counseling as an integrated, routine aspect of care. Although physicians traditionally have focused their clinical efforts on cancer therapy, they also have a crucial role in cancer prevention through the identification of individuals with hereditary cancer syndromes.

\section{Acknowledgments}

Research support: NIH T32 \#CA101642, NCI P30 \#CA16672. 


\section{References}

1. Miki Y, Swensen J, Shattuck-Eidens D, Futreal PA, Harshman K, Tavtigian S, et al. A strong candidate for the breast and ovarian cancer susceptibility gene BRCA1. Science. 1994; 266:66-71. [PubMed: 7545954]

2. Wooster R, Bignell G, Lancaster J, Swift S, Seal S, Mangion J, et al. Identification of the breast cancer susceptibility gene BRCA2. Nature. 1995; 378:789-92. [PubMed: 8524414]

3. Antoniou AC, Gayther SA, Stratton JF, Ponder BA, Easton DF. Risk models for familial ovarian and breast cancer. Genet Epidemiol. 2000; 18:173-90. [PubMed: 10642429]

4. Peto J, Collins N, Barfoot R, Seal S, Warren W, Rahman N, et al. Prevalence of BRCA1 and BRCA2 gene mutations in patients with early-onset breast cancer. J Natl Cancer Inst. 1999; 91:9439. [PubMed: 10359546]

5. Anglian Breast Cancer Study Group. Prevalence and penetrance of BRCA1 and BRCA2 mutations in a population-based series of breast cancer cases. Br J Cancer. 2000; 83:1301-8. [PubMed: 11044354]

6. Claus EB, Schildkraut JM, Thompson WD, Risch NJ. The genetic attributable risk of breast and ovarian cancer. Cancer. 1996; 77:2318-24. [PubMed: 8635102]

7. Pal T, Permuth-Wey J, Betts JA, Krischer JP, Fiorica J, Arango H, et al. BRCA1 and BRCA2 mutations account for a large proportion of ovarian carcinoma cases. Cancer. 2005; 104:2807-16. [PubMed: 16284991]

8. Risch HA, McLaughlin JR, Cole DE, Rosen B, Bradley L, Kwan E, et al. Prevalence and penetrance of germline BRCA1 and BRCA2 mutations in a population series of 649 women with ovarian cancer. Am J Hum Genet. 2001; 68:700-10. [PubMed: 11179017]

9. Couch FJ, DeShano ML, Blackwood MA, Calzone K, Stopfer J, Campeau L, et al. BRCA1 mutations in women attending clinics that evaluate the risk of breast cancer. N Engl J Med. 1997; 336:1409-15. [PubMed: 9145677]

10. Frank TS, Manley SA, Olopade OI, Cummings S, Garber JE, Bernhardt B, et al. Sequence analysis of BRCA1 and BRCA2: correlation of mutations with family history and ovarian cancer risk. J Clin Oncol. 1998; 16:2417-25. [PubMed: 9667259]

11. Parmigiani G, Berry D, Aguilar O. Determining carrier probabilities for breast cancersusceptibility genes BRCA1 and BRCA2. Am J Hum Genet. 1998; 62:145-58. [PubMed: 9443863]

12. Shattuck-Eidens D, Oliphant A, McClure M, McBride C, Gupte J, Rubano T, et al. BRCA1 sequence analysis in women at high risk for susceptibility mutations. Risk factor analysis and implications for genetic testing. JAMA. 1997; 278:1242-50. [PubMed: 9333265]

13. National Comprehensive Cancer Network. Genetic/familial high-risk assessment of breast and ovarian clinical practice guidelines in oncology. Available at: http://www.nccn.org/professionals/ physician_gls/PDF/genetics_screening.pdf. Retrieved March 9, 2009

14. Lancaster JM, Powell CB, Kauff ND, Cass I, Chen LM, Lu KH, et al. Society of gynecologic oncologists education committee statement on risk assessment for inherited gynecologic cancer predispositions. Gynecol Oncol. 2007; 107:159-62. [PubMed: 17950381]

15. American College of Obstetricians and Gynecologists. Hereditary breast and ovarian cancer syndrome. ACOG Practice Bulletin No. 103. Obstet Gynecol. 2009; 113:957-66. [PubMed: 19305347]

16. Guillem JG, Wood WC, Moley JF, Berchuck A, Karlan BY, Mutch DG, et al. ASCO/SSO review of current role of risk-reducing surgery in common hereditary cancer syndromes. J Clin Oncol. 2006; 24:4642-60. [PubMed: 17008706]

17. Lee SC, Bernhardt BA, Helzlsouer KJ. Utilization of BRCA1/2 genetic testing in the clinical setting: report from a single institution. Cancer. 2002; 94:1876-85. [PubMed: 11920551]

18. Lynch HT, Boland CR, Rodriguez-Bigas MA, Amos C, Lynch JF, Lynch PM. Who should be sent for genetic testing in hereditary colorectal cancer syndromes? J Clin Oncol. 2007; 25:3534-42. [PubMed: 17687158] 
19. Rubin SC, Benjamin I, Behbakht K, Takahashi H, Morgan MA, LiVolsi VA, et al. Clinical and pathological features of ovarian cancer in women with germ-line mutations of BRCA1. N Engl J Med. 1996; 335:1413-6. [PubMed: 8875917]

20. Boyd J, Sonoda Y, Federici MG, Bogomolniy F, Rhei E, Maresco DL, et al. Clinicopathologic features of BRCA-linked and sporadic ovarian cancer. JAMA. 2000; 283:2260-5. [PubMed: 10807385]

21. Ben David Y, Chetrit A, Hirsh-Yechezkel G, Friedman E, Beck BD, Beller U, et al. Effect of BRCA mutations on the length of survival in epithelial ovarian tumors. J Clin Oncol. 2002; 20:463-6. [PubMed: 11786575]

22. Pal T, Permuth-Wey J, Kapoor R, Cantor A, Sutphen R. Improved survival in BRCA2 carriers with ovarian cancer. Fam Cancer. 2007; 6:113-9. [PubMed: 17160431]

23. Tan DS, Rothermundt C, Thomas K, Bancroft E, Eeles R, Shanley S, et al. "BRCAness" syndrome in ovarian cancer: a case-control study describing the clinical features and outcome of patients with epithelial ovarian cancer associated with BRCA1 and BRCA2 mutations. J Clin Oncol. 2008; 26:5530-6. [PubMed: 18955455]

24. Kieran S, Loescher LJ, Lim KH. The role of financial factors in acceptance of clinical BRCA genetic testing. Genet Test. 2007; 11:101-10. [PubMed: 17394399]

25. Chaliki H, Loader S, Levenkron JC, Logan-Young W, Hall WJ, Rowley PT. Women's receptivity to testing for a genetic susceptibility to breast cancer. Am J Public Health. 1995; 85:1133-5. [PubMed: 7625512]

26. Durfy SJ, Bowen DJ, McTiernan A, Sporleder J, Burke W. Attitudes and interest in genetic testing for breast and ovarian cancer susceptibility in diverse groups of women in western Washington. Cancer Epidemiol Biomarkers Prev. 1999; 8:369-75. [PubMed: 10207642]

27. Lerman C, Narod S, Schulman K, Hughes C, Gomez-Caminero A, Bonney G, et al. BRCA1 testing in families with hereditary breast-ovarian cancer. A prospective study of patient decision making and outcomes. JAMA. 1996; 275:1885-92. [PubMed: 8648868]

28. Geer KP, Ropka ME, Cohn WF, Jones SM, Miesfeldt S. Factors influencing patients' decisions to decline cancer genetic counseling services. J Genet Counseling. 2001; 10:25-40.

29. Centers for Medicare and Medicaid Services. LCD for genetic testing (L24308). Available at: http://www.cms.hhs.gov/mcd/viewlcd.asp?lcd_id=24308\&lcd_version=2\&show=all. Retrieved October 29, 2007

30. Lacour RA, Daniels MS, Westin SN, Meyer LA, Burke CC, Burns KA, et al. What women with ovarian cancer think and know about genetic testing. Gynecol Oncol. 2008; 111:132-6. [PubMed: 18684498] 


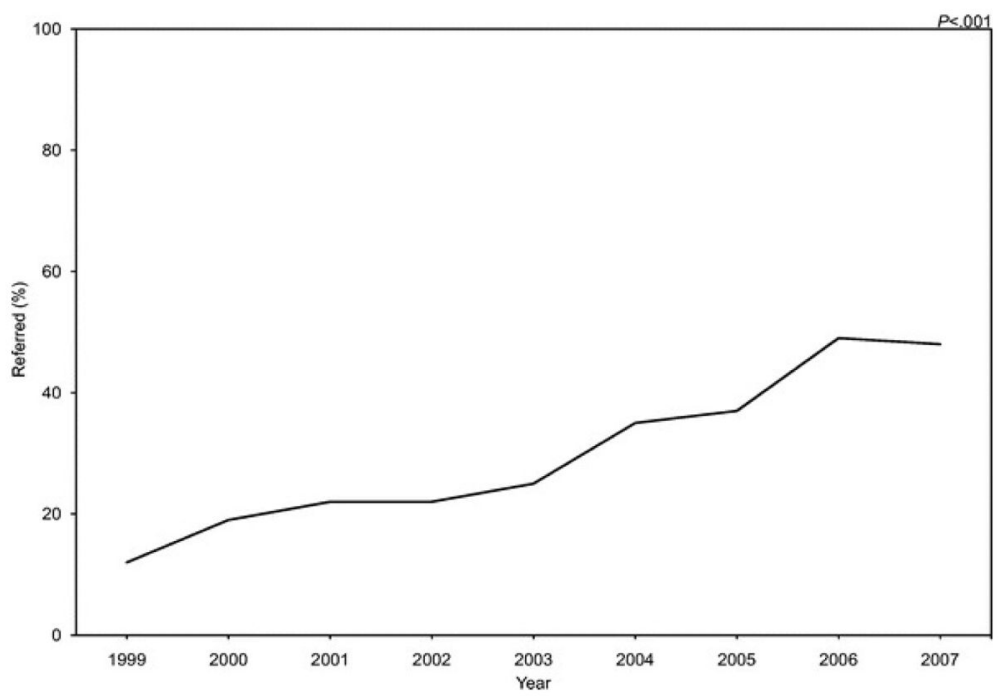

Fig. 1.

Proportion of patients meeting substantial (more than 20-25\%) risk criteria for a $B R C A 1$ or $B R C A 2$ mutation referred for genetic counseling by study year. $P<.001$ by Fisher's exact test. 


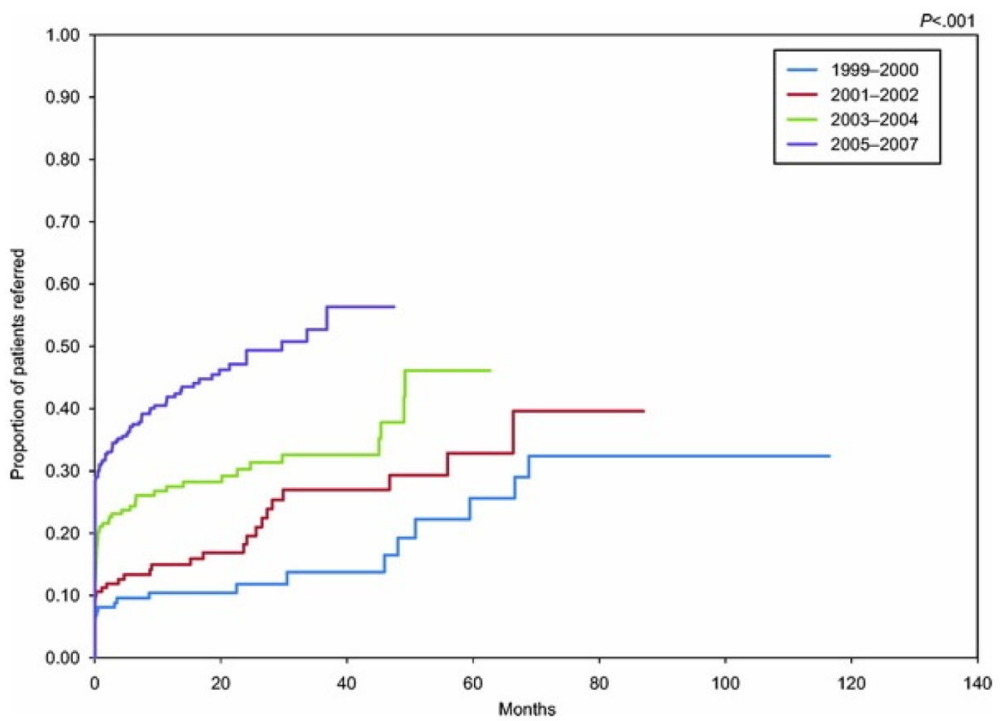

Fig. 2.

Kaplan-Meier curve of time to genetic counseling referral for patients meeting substantial (more than 20-25\%) risk criteria for a $B R C A 1$ or $B R C A 2$ mutation. $P<.001$ by log-rank test. 


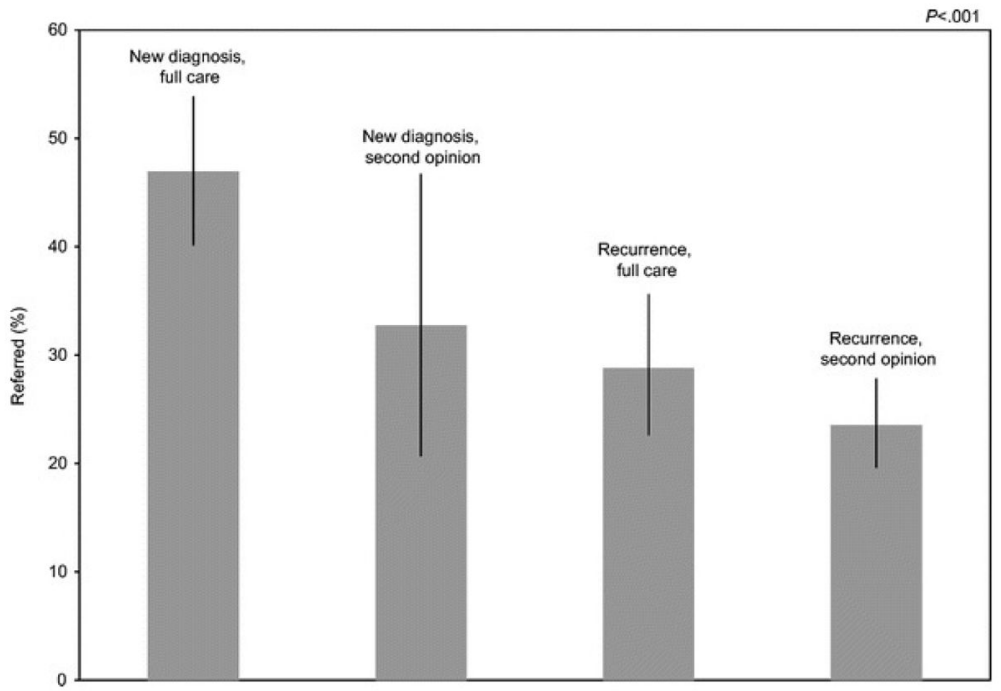

Fig. 3.

Proportion of patients meeting substantial (more than 20-25\%) risk criteria for a $B R C A 1$ or $B R C A 2$ mutation who were referred for genetic counseling by patient type. 


\section{Table 1}

\section{Demographics and Clinical Characteristics}

\begin{tabular}{|c|c|c|}
\hline Variable & $\mathbf{n}$ & $\%$ \\
\hline Age (y) (mean \pm standard deviation) & $56.37 \pm 11.80$ & \\
\hline \multicolumn{3}{|l|}{ Site of cancer } \\
\hline Ovary & 3,569 & 94.89 \\
\hline Fallopian tube & 140 & 3.72 \\
\hline Primary peritoneal & 50 & 1.33 \\
\hline Müllerian-not otherwise specified & 2 & 0.05 \\
\hline \multicolumn{3}{|l|}{ Histology } \\
\hline Serous & 1,981 & 52.62 \\
\hline Endometrioid & 178 & 4.73 \\
\hline Clear cell & 213 & 5.66 \\
\hline Mixed & 995 & 26.43 \\
\hline Mucinous & 59 & 1.57 \\
\hline Other histology/unspecified adenocarcinoma & 339 & 9.00 \\
\hline \multicolumn{3}{|l|}{ Stage } \\
\hline I & 316 & 8.39 \\
\hline II & 219 & 5.82 \\
\hline III & 2,461 & 65.37 \\
\hline IV & 422 & 11.21 \\
\hline Unknown & 347 & 9.22 \\
\hline \multicolumn{3}{|l|}{ Grade } \\
\hline 1 & 335 & 8.90 \\
\hline 2 & 196 & 5.21 \\
\hline 3 & 2,908 & 77.24 \\
\hline Unknown & 326 & 8.66 \\
\hline \multicolumn{3}{|l|}{ Race } \\
\hline White & 3,110 & 82.60 \\
\hline African American & 155 & 4.12 \\
\hline Hispanic & 360 & 9.56 \\
\hline Other & 133 & 3.53 \\
\hline Unknown & 7 & 0.19 \\
\hline \multicolumn{3}{|l|}{ Jewish } \\
\hline Not Jewish & 3,323 & 88.26 \\
\hline Jewish, Ashkenazi ancestry & 18 & 0.48 \\
\hline Jewish, not otherwise specified & 143 & 3.80 \\
\hline Unknown & 281 & 7.46 \\
\hline \multicolumn{3}{|l|}{ Type of patient } \\
\hline New diagnosis, full care & 968 & 25.71 \\
\hline New diagnosis, second opinion & 298 & 7.92 \\
\hline Recurrence, full care & 747 & 19.84 \\
\hline
\end{tabular}




\begin{tabular}{lcr}
\hline Variable & $\mathbf{n}$ & $\boldsymbol{\%}$ \\
\hline Recurrence, second opinion & 1,748 & 46.43 \\
Unknown & 4 & 0.11 \\
\hline
\end{tabular}


Table 2

Proportion of Ovarian Cancer Patients Meeting Referral Criteria

\begin{tabular}{lr}
\hline Number of Patients per Year & Number $(\%)$ of Patients at Substantial (More Than 20-25\%) Risk for a BRCA1 or BRCA2 Mutation \\
\hline 1999: 361 & $74(20.50)$ \\
2000: 420 & $89(21.19)$ \\
2001: 387 & $78(20.16)$ \\
2002: 444 & $93(20.95)$ \\
2003: 425 & $113(26.59)$ \\
2004: 463 & $125(27.00)$ \\
2005: 466 & $134(28.76)$ \\
2006: 380 & $81(21.32)$ \\
2007: 419 & $109(26.01)$ \\
Total 3,765 & $896(23.80)$ \\
\hline
\end{tabular}


Table 3

Logistic Regression-Reduced Model to Predict Patient Referral for Genetic Counseling (Including Only Those Patients Who Met Substantial (More Than $20-25 \%$ ) Risk for a BRCA1 or BRCA2 Mutation)

\begin{tabular}{lcrc}
\hline Variable & Odds Ratio & $\boldsymbol{P}$ & 95\% Confidence Interval \\
\hline Personal history of breast cancer & 3.63 & $<.001$ & $2.27-5.83$ \\
Family history of breast cancer age 50 or younger or ovarian cancer at any age & 1.83 & .021 & $1.09-3.07$ \\
Year of initial visit & 1.28 & $<.001$ & $1.17-1.40$ \\
Age at initial visit & 0.97 & $<.001$ & $0.95-0.99$ \\
Patient type (reference: new diagnosis, full care) & & & \\
$\quad$ New diagnosis, second opinion & 0.47 & .061 & $0.22-1.03$ \\
Recurrence, full care & 0.44 & .001 & $0.27-0.72$ \\
$\quad$ Recurrence, second opinion & 0.23 & $<.001$ & $0.15-0.35$ \\
Grade (reference: 1) & & & \\
2 & 1.59 & .445 & $0.48-5.24$ \\
3 & 2.98 & .005 & $1.39-6.38$ \\
Jewish & 2.51 & .004 & $1.33-4.73$ \\
Race (reference: white) & & & \\
$\quad$ African American & & & \\
Hispanic & 0.25 & .009 & $0.09-0.70$ \\
\hline
\end{tabular}

